\title{
Short-term photoacclimation and photoregulation strategies of Sargassum horneri in response to temperature and light
}

\author{
Z.H. ZHONG*,†, Y. WANG ${ }^{*, *, \dagger}$, S. QIN", L.C. ZHUANG*, J.J. LI**, W.L. SONG ${ }^{*,+}$, and Z.Y. LIU ${ }^{*,+}$ \\ Yantai Institute of Coastal Zone Research, Chinese Academy of Sciences, 264003 Yantai, China* \\ Hohai University, Nanjing, 210098 Jiangsu, China**
}

\begin{abstract}
Sargassum horneri (Turner) C. Agardh is a genus of brown algae and plays an important role in marine ecosystem. However, the inhabiting area of $S$. horneri has been decreasing sharply in China. To understand the photoacclimation and photoregulation strategies of $S$. horneri in responses to temperature and light, S. horneri was cultured under different temperatures $\left[18^{\circ} \mathrm{C}\right.$ (LT) and $\left.26^{\circ} \mathrm{C}(\mathrm{HT})\right]$ and light intensities $\left[60 \mu \mathrm{mol}\left(\right.\right.$ photon) $\mathrm{m}^{-2} \mathrm{~s}^{-1}$ (LL) and $120 \mu \mathrm{mol}\left(\right.$ photon) $\mathrm{m}^{-2} \mathrm{~s}^{-1}$ (HL)] for $7 \mathrm{~d}$, and then the chlorophyll $a$ fluorescence parameters were measured. The results showed that the maximum electron transfer rate occurred at low temperature and high light (LT-HL) condition. The high temperature was the predominant factor for causing inhibition of PSII, lowering the effective quantum yield of PSII, and reducing the nonphotochemical quenching (NPQ). However, high light could improve the photoprotective ability via enhancing the NPQ. On the other hand, a strong linear relationship was observed between NPQ and the electron transport efficiency $(\alpha)$; the increase of NPQ could reduce the $\alpha$ value and avoid damage from high light stress to PSII. Therefore, S. horneri was found to be well adapted to grow under LT-HL conditions.
\end{abstract}

Keywords: photoacclimation; photoprotective ability; rapid light-response curves; Sargassum horneri; steady-state light-response curve.

\section{Highlights}

- High temperature damages PSII of Sargassum horneri

- High light improves the photoprotective ability in $S$. horneri

- $S$. horneri is adapted to grow under low temperature and high light conditions
Received 27 October 2020

Accepted 22 March 2021

Published online 23 April 2021

${ }^{+}$Corresponding authors

e-mail: wlsong@yic.ac.cn zyliu@yic.ac.cn

\section{Introduction}

Sargassum horneri (Turner) C. Agardh is a genus of brown algae (Fucales, Phaeophyta) inhabiting rocky coasts along the northwest coast of the Pacific (Komatsu et al. 1982) in subtidal zone (3-4 $\mathrm{m}$ below the low tidal line) (Sun et al. 2010). Under normal conditions, it can form a flourishing forest in spring and provide a habitat for spawning, nursing, and feeding for marine organisms (Miki et al. 2016). On the other hand, Sargassum forest is an effective biofilter to purify effluent waters, playing an important role in marine ecosystems (Pang et al. 2009). Besides, $S$. horneri is often consumed in Asian countries as a bio-functional material source of fucoxanthin and other

Abbreviations: $\mathrm{Chl}$ - chlorophyll; $\mathrm{F}_{0}$ - minimal fluorescence yield of the dark-adapted state; $\mathrm{F}_{\mathrm{m}}$ - maximal fluorescence yield of the darkadapted state; $\mathrm{F}_{\mathrm{m}}{ }^{\prime}-$ maximal fluorescence yield of the light-adapted state; $\mathrm{F}_{\mathrm{s}}$ - steady-state fluorescence yield; $\mathrm{F}_{\mathrm{v}}-$ variable fluorescence; $\mathrm{F}_{\mathrm{v}} / \mathrm{F}_{\mathrm{m}}$ - maximum PSII quantum yield; $I_{\mathrm{k}}$ - saturated irradiance; $\mathrm{LC}$ - steady-state light curve; NPQ - nonphotochemical quenching; RCII - PSII reaction center; rETR - relative electron transport rate; $\mathrm{rETR}_{\max }$ - maximum electron transport rate; RLC - rapid light curve; $\mathrm{Y}_{(\mathrm{II})}$ - actual photochemical efficiency of PSII; $\mathrm{Y}_{(\mathrm{NO})}$ - quantum yield of nonregulated energy dissipation; $\mathrm{Y}_{(\mathrm{NPQ})}-$ quantum yield of regulated energy dissipation; $\alpha$ - electron transport efficiency.

Acknowledgements: This work was supported by Science and Technology Innovation Development Planning of Yantai (2020MSGY058), the National Key R\&D Program of China (2017YFC0506200), Shandong Province Science Foundation for Youths (ZR201807120023), the Key Research and Development Program of Yantai City (No. 2018ZHGY082), and the Science and Technology Innovation Development Planning of Yantai City (2020MSGY068 and 2020MSGY055).

Conflict of interest: The authors declare that they have no conflict of interest.

The authors contributed equally to this work. 
bioactive compounds (Murakami et al. 2011, Sanjeewa et al. 2017). However, the natural Sargassum forest has been degenerating in recent years due to the following reasons: (1) land reclamation (Terawaki et al. 2003); (2) the grazing damage from herbivores, such as sea urchins, abalone, and holothurians (Zhang et al. 2008); and (3) the deterioration of marine environment, elevated temperature (Komatsu et al. 2014), limited transparency (Sun et al. 2010), misbalanced nutrition (Zhang et al. 2008), and increased pollution (Yu et al. 2019).

In China, Sargassum spp. were the dominant species in intertidal and subtidal zones in Gouqi Island, Zhejiang, China, where over $90 \%$ of the total biomass were $S$. horneri (Zhang et al. 2008). From 1960 to 1965, distribution of $S$. horneri was widespread although its abundance was lower than local dominant species (Sun et al. 2010). In the recent decade, the area of $S$. horneri beds has been shrinking quickly in China (Yu et al. 2019), especially, in Nanji Islands, Zhejiang, China from 1980 to 1985. Although the distribution of $S$. horneri was wide, its abundance was very low. From 2000 to 2007, S. horneri almost disappeared (Sun et al. 2010). The causes were most probably the elevated temperature (Zhang et al. 2008) and the limited light (Sun et al. 2010, Bi et al. 2014).

It was reported that the global sea surface temperature has been considerably increasing at a rate of $\sim 0.12^{\circ} \mathrm{C}$ per decade in recent 30 years (IPCC 2013). The predicted ocean warming posed a threat to the survival of $S$. horneri; the southern limit of $S$. horneri distribution would move northward and may disappear in 2100 (Komatsu et al. 2014). The elevated ocean temperature was known to affect the primary productivity by directly or indirectly altering algal physiological performance (Gao et al. 2017). Photosynthesis is highly sensitive to high temperature and often inhibited before other cellular functions are impaired. Photosystem II (PSII) and ribulose-1,5-bisphosphate carboxylase/oxygenase (Rubisco) are the major attacked targets; high temperature can damage PSII and reduce the Rubisco activity by inactivating the Rubisco activase (Koch et al. 2013). Additionally, sensitivity of other main cellular components is directly subject to high temperature (Harley et al. 2012). The membrane properties and intracellular milieu could be changed by high temperature and thus cell functions are impaired. An increase in fluidity of thylakoid membranes caused by high temperature could dislodge PSII light harvesting (Mathur et al. 2014).

In addition, subtidal macroalgae are sensitive to the excess and fluctuating light (Li et al. 2014). In a shallow coastal zone, light could fluctuate on different time scales (from seconds to days) in predictable (day length, tidal period, and solar angle) or unpredictable (sunshine, cloud, turbidity, and rain) manner (Williamson et al. 2018). Excess or fluctuating light may result in photoinhibition and the accumulation of reactive oxygen species (ROS) in photosynthetic apparatus, especially the PSII. Protein D1 is one of sensitive targets to photodamage. When protein $\mathrm{D} 1$ is damaged, it will be removed and replaced by de novo synthesized proteins during the D1 repairing cycle. When the rate of photodamage exceeds the D1 repair capacity, photoinhibition will take place. The imbalance between energy absorption and consumption can lead to production of ROS and damage the membrane fluidity of the thylakoid membranes, while D1 repairing cycle is tightly dependent on the membrane fluidity of the thylakoid membranes (Yamamoto 2016).

Being exposed to the changing environmental stress, macroalgae must optimize their photosynthetic performance through photoacclimation. Otherwise, the photodamage to the photosynthetic system would occur, resulting in the markedly inhibition of photosynthesis (Müller et al. 2001). Photoacclimation (hours to days) is a phenotypic acclimation in response to environmental conditions through changing the size or number of photosynthetic units (Williamson et al. 2018). While under a sudden environmental stress, photoregulation (seconds to minutes) is needed to guarantee the safe dissipation of surplus absorbed energy as heat to prevent photodamage to the photosynthetic system (Lavaud and Lepetit 2013). In addition, nonphotochemical quenching (NPQ) is one of the most important photoprotective mechanisms for phototropic organisms (Ocampo-Alvarez et al. 2013). By the help of the xanthophyll cycle (XC) during NPQ, excess absorbed energy is dissipated as heat (Esteban et al. 2009, García-Mendoza et al. 2011, Ocampo-Alvarez et al. 2013). Thus, for macroalgae, photoacclimation and photoregulationarehigh-efficiencystrategies toadjustphotosynthetic performances to multiple environmental stresses.

The pulse amplitude modulated (PAM) fluorometry is an efficient technology to measure chlorophyll (Chl) $a$ fluorescence, which includes rich information about photosynthetic apparatus (Stirbet and Govindjee 2011). The evident advantage of PAM is instantaneous and nondestructive measurement of real-time activity of photosynthesis (White and Critchley 1999). One technique of PAM is using the rapid light-responses curves (RLC), allowing to rapidly $(<2 \mathrm{~min})$ detect the adjustments in photosynthetic performance(short-term photoacclimation), related mainly to the carbon metabolism, photoprotective mechanism, and photoinhibition (Ralph and Gademann 2005). In opposition to RLC, the steady-state light-response curves (LC) characterize the long-term photoacclimation status. LC represents the potential response to long-term light exposure conditions, while the RLC aims at the acclimation status to recent light history (Serôdio et al. 2006). Discrepancy between RLC and LC was shown in the nonphotochemical quenching (NPQ) processes, during which extra absorbed light energy could be dissipated. RLC and LC have been successfully applied to study the photosynthetic performance of benthic diatom and provide an effective way to detect the photoacclimation of other algae. At present, however, details of the inter-action between seawater temperature and light on the photosynthetic performance of $S$. horneri remain poorly understood.

In the present study, the short-term photoacclimation and photoregulation strategies of $S$. horneri in responses to temperature and light were investigated. We hope that the results may provide an insight to photosynthetic characteristics of $S$. horneri and a support to the protection of Sargassum forest. 


\section{Materials and methods}

Materials: S. horneri were sampled in a subtidal Sargassum forest in Changdao Island $\left(38^{\circ} 21^{\prime} 22.48^{\prime \prime} \mathrm{N}\right.$, $\left.120^{\circ} 54^{\prime} 30.78^{\prime \prime} \mathrm{E}\right)$, Shandong Province, China, in September 2019. The thalli were washed and delivered to laboratory in cooler box in $48 \mathrm{~h}$. After arriving to laboratory, algae were further cleaned with sterile seawater gently to remove debris and epiphytes and cultured in water tank in $200 \mathrm{~L}$ of sterile natural seawater enriched with $100 \mu \mathrm{M} \mathrm{NaNO}$ and $10 \mu \mathrm{M} \mathrm{KH} \mathrm{KO}_{4}$. The culture medium was bubbled with ambient air and refreshed daily; light intensity was set as $120 \mu \mathrm{mol}$ (photon) $\mathrm{m}^{-2} \mathrm{~s}^{-1}$ in photoperiod of $12 \mathrm{~h}(\mathrm{~L}): 12 \mathrm{~h}(\mathrm{D})$.

Experimental design: To investigate the effects of temperature and light on $S$. horneri growth, approximately $0.1 \mathrm{~g}$ of thalli fresh mass (FM) were incubated in $500-\mathrm{mL}$ flasks, then grown at two temperatures $\left(18\right.$ and $\left.26^{\circ} \mathrm{C}\right)$ and two light intensities [60 and $120 \mu \mathrm{mol}$ (photon) $\mathrm{m}^{-2} \mathrm{~s}^{-1}$ ] in photoperiod of $12 \mathrm{~h}(\mathrm{~L}): 12 \mathrm{~h}(\mathrm{D})$ (triplicate for each treatment). The medium was sterile natural seawater enriched with $\mathrm{f} / 2$, gently bubbled with ambient air, and renewed daily. The chosen temperature of $18^{\circ} \mathrm{C}$ was the optimal growth temperature of S. horneri (Sun et al. 2008), while that of $26^{\circ} \mathrm{C}$ was the upper threshold of $S$. horneri survival (Yu et al. 2019). The chosen light intensities were low but higher than the light-compensation point to provide a high light intensity range above the saturating irradiance [100 $\mu$ mol(photon) $\mathrm{m}^{-2} \mathrm{~s}^{-1}$, which was close to the maximum midday light intensity at the sample collection site. Chl $a$ fluorescence parameters were measured after being cultured for $7 \mathrm{~d}$.

Chl $\boldsymbol{a}$ fluorescence parameters: The fluorescence parameters were measured with a pulse amplitude modulated fluorometer (DIVING-PAM, Walz, Effeltrich, Germany). All measurements were made with dark leaf clip (DIVING-LC) plus adapter positioning fiber optics tips perpendicularly above the algae at a constant distance of $3 \mathrm{~mm}$. Samples were placed in natural seawater at a culture temperature controlled by air conditioner. After 30-min dark adaption under the same conditions as the culture environment (except for light), the rapid light curves (RLC) and steady-state light curves (LC) were measured according to the protocol of Serôdio et al. (2006). After dark adaptation, $F_{0}$ and $F_{m}$ were measured using the saturating pulses $\left[3,577 \mu \mathrm{mol}\right.$ (photon) $\mathrm{m}^{-2} \mathrm{~s}^{-1}$ for $800 \mathrm{~ms}$. RLC was created by exposing samples to eight increasing levels of light intensity [38, 104, 186, 322, 463, 621, 893; and 1,189 $\mu \mathrm{mol}$ (photon) $\mathrm{m}^{-2} \mathrm{~s}^{-1}$ ] for $10 \mathrm{~s}$. At each actinic light, $F$ and $F_{m}{ }^{\prime}$ were determined every $90 \mathrm{~s}$ until the steady state was achieved (about 4.5-15 min). An RLC was constructed immediately after each light level of the LC and was termed the steady-state rapid light curves (SRLC). After the completion of the SRLC, the sample was subjected to the next LC light level and allowed to reach a new steady state.

The following parameters were calculated based on the studies of Bilger and Björkman (1990), Hendrickson et al.
(2004), and Belshe et al. (2007):

Maximum PSII quantum yield:

$\mathrm{F}_{\mathrm{v}} / \mathrm{F}_{\mathrm{m}}=\left(\mathrm{F}_{\mathrm{m}}-\mathrm{F}_{0}\right) / \mathrm{F}_{\mathrm{m}}$

Effective quantum yield of PSII:

$\mathrm{Y}_{(\mathrm{II})}=\mathrm{F}_{\mathrm{v}}{ }^{\prime} / \mathrm{F}_{\mathrm{m}}{ }^{\prime}=\left(\mathrm{F}_{\mathrm{m}}{ }^{\prime}-\mathrm{F}_{\mathrm{s}}\right) / \mathrm{F}_{\mathrm{m}}{ }^{\prime}$

Nonphotochemical quenching:

$\mathrm{NPQ}=\left(\mathrm{F}_{\mathrm{m}}-\mathrm{F}_{\mathrm{m}}{ }^{\prime}\right) / \mathrm{F}_{\mathrm{m}}{ }^{\prime}$

Quantum yield of regulated energy dissipation:

$\mathrm{Y}_{(\mathrm{NPQ})}=\left(\mathrm{F}_{\mathrm{s}} / \mathrm{F}_{\mathrm{m}}{ }^{\prime}\right)-\left(\mathrm{F}_{\mathrm{s}} / \mathrm{F}_{\mathrm{m}}\right)$

Quantum yield of nonregulated energy dissipation:

$\mathrm{Y}_{(\mathrm{NO})}=\mathrm{F}_{\mathrm{s}} / \mathrm{F}_{\mathrm{m}}{ }^{\prime}$

The relative electron transfer rate:

$\mathrm{rETR}=\left(\mathrm{F}_{\mathrm{v}}{ }^{\prime} \times \mathrm{AF} \times \mathrm{PAR} \times 0.5\right) / \mathrm{F}_{\mathrm{m}}{ }^{\prime}$

where AF is the light absorption capacity that used the most common value 0.84 ; PAR is the actinic photosynthetically active radiation; and 0.5 is the relative distribution of absorbed energy to PSII. The parameters of the RLC and LC were calculated from the rETR curves following the models (Jassby and Platt 1976):

$\mathrm{rETR}=\mathrm{rETR}_{\max } \times \tanh \frac{\alpha \times I}{\mathrm{rETR}_{\max }}$

$I_{\mathrm{k}}=\mathrm{rETR}_{\max } / \alpha$

where $\mathrm{rETR}_{\max }$ is the light-saturated electron transport rate; $\alpha$ is the light-harvesting efficiency; $I$ is the incident irradiance; $I_{\mathrm{k}}$ is saturated irradiance.

According to Serôdio and Lavaud (2011), the fitting formula of NPQ $v s$. PAR is:

$\mathrm{NPQ}=\frac{\mathrm{NPQ} \max \times I^{\mathrm{n}}}{I_{50}{ }^{\mathrm{n}}+I^{\mathrm{n}}}$

where $\mathrm{NPQ}_{\max }$ is the maximum NPQ value; $I_{50}$ is the irradiance level corresponding to $50 \%$ of $\mathrm{NPQ}_{\max }$, and $n$ is the sigmoidicity coefficient.

Statistical analyses: Significance of differences between treatments was tested using one-way or two-way analysis of variance (ANOVA, Tukey's post-hoc test) using the SPSS 24.0. $P<0.05$ was considered significant. All data were expressed as the mean \pm standard deviation (SD, $n \geq 3$ ).

\section{Results}

$\mathbf{F}_{\mathrm{v}} / \mathbf{F}_{\mathrm{m}}$ variation with temperature and light: The $F_{\mathrm{v}} / \mathrm{F}_{\mathrm{m}}$ ratio decreased with the increase of temperature and light (Fig. 1). The maximum $F_{v} / F_{m}$ value of 0.7288 occurred at low temperature and low light (LT-LL), while the $\mathrm{F}_{\mathrm{v}} / \mathrm{F}_{\mathrm{m}}$ dropped to 0.7053 under high temperature and high light (HT-HL), the difference between them was significant. Two-way ANOVA showed that temperature and light were the main factors causing the decrease of $\mathrm{F}_{\mathrm{v}} / \mathrm{F}_{\mathrm{m}}$. However, temperature and light had no significant interactive effect on $\mathrm{F}_{\mathrm{v}} / \mathrm{F}_{\mathrm{m}}$ (Table 1). 


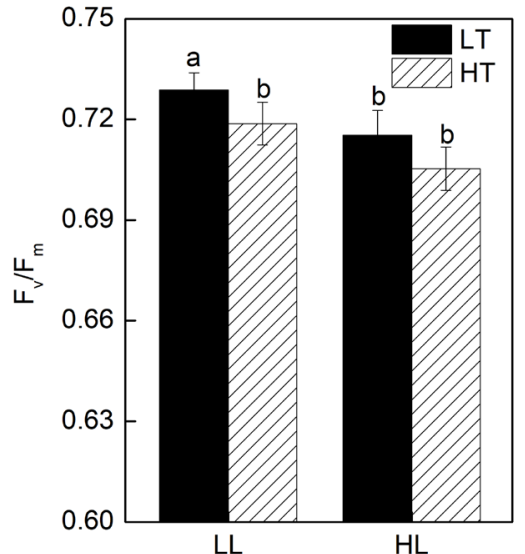

Fig. 1. The maximum quantum yield $\left(\mathrm{F}_{\mathrm{v}} / \mathrm{F}_{\mathrm{m}}\right)$ measured in Sargassum horneri cultured at different light and temperature. LT - low temperature; HT - high temperature; LL - low light; $\mathrm{HL}$ - high light. Values are means $\pm \mathrm{SD}, n=3$. Different letters above the bars indicate significant differences $(P<0.05)$ between the different treatments.

rETR and NPQ vs. PAR curves: After growth under different temperature and light conditions for $7 \mathrm{~d}$, the relationship of rapid light curves (RLC) and steady-state light curves (LC) to temperature and light was significantly different (Fig. 2). The photoacclimation status could be inferred from the photosynthetic parameters (Table 2). In the RLC, the high light decreased the electron transport efficiency $(\alpha)$, and the differences were not significant compared to that of the low light, whilst under the high light, the $\alpha$ in the LC showed no change. Besides, the differences between all $\alpha$ values estimated from RLC and LC were not significant. Comparison in $\mathrm{rETR}_{\max }$ of the $S$. horneri samples revealed that $\mathrm{rETR}_{\max }$ in $\mathrm{LC}$ was higher than that in RLC under the same treatment conditions. On the other hand, the rETR $\mathrm{max}_{\max }$ estimated from RLC was not obviously different between those of the LT-LL, LT-HL, and HT-LL, but all of them were significantly higher than that of HT-HL in a range from 26 to $40 \%$. Two-way ANOVA showed that temperature and light had an interactive effect and both temperature and light exerted a main effect on the $\mathrm{rETR}_{\max }$. As shown in Table 2, the high light boosted the inhibition to the $\mathrm{rETR}_{\max }$ by the high temperature. Conversely, the increase of light did not significantly alter the $\mathrm{rETR}_{\max }$ in $\mathrm{LC}$ under the high temperature, whilst high light obviously increased the $\mathrm{rETR}_{\max }$ under the low temperature. Regarding $I_{\mathrm{k}}$, the patterns of responses to temperature and light in RLC and LC were generally the same as the $\mathrm{rETR}_{\max }$. In RLC, the maximum and minimum $I_{\mathrm{k}}$ was observed at LT-HL and

Table 1. Two-way analysis of variance in the effects of temperature and light on maximum PSII quantum yield $\left(\mathrm{F}_{\mathrm{v}} / \mathrm{F}_{\mathrm{m}}\right)$, maximum electron transport rate of a rapid light curve $\left[\mathrm{ETR}_{\max (\mathrm{RLC})}\right]$, electron transport efficiency of the initial slope of a rapid light curve $\left(\alpha_{\mathrm{RLC}}\right)$, saturated irradiance of a rapid light curve $\left[I_{\mathrm{k}(\mathrm{RLC})}\right]$, nonphotochemical quenching of a rapid light curve $\left(\mathrm{NPQ}_{\mathrm{RLC}}\right)$, maximum electron transport rate of a steady-state light curve $\left[\mathrm{ETR}_{\max (\mathrm{RLC})}\right]$, electron transport efficiency of the initial slope of a steady-state light curve $\left(\alpha_{\mathrm{LC}}\right)$, saturated irradiance of a steady-state light curve $\left[I_{\mathrm{k}(\mathrm{LC})}\right]$, and nonphotochemical quenching of a steady-state light curve $\left(\mathrm{NPQ} \mathrm{LC}_{\mathrm{LC}}\right)$ of Sargassum horneri. Temperature $\times$ light represents the interactive effect between these two factors; df represents degrees of freedom; and $F$ represents the value of the $F$ statistic.

\begin{tabular}{|c|c|c|c|c|c|c|c|}
\hline Source & df & $F$ & $P$ value & Source & df & $F$ & $P$ value \\
\hline $\mathrm{F}_{\mathrm{v}} / \mathrm{F}_{\mathrm{m}}$ & & & & $\mathrm{ETR}_{\max (\mathrm{RLC})}$ & & & \\
\hline Temperature & 1 & 14.586 & $<0.05$ & Temperature & 1 & 11.43 & $<0.05$ \\
\hline Light & 1 & 19.651 & $<0.05$ & Light & 1 & 8.97 & $<0.05$ \\
\hline Temperature $\times$ light & 1 & 0.228 & $>0.05$ & Temperature $\times$ light & 1 & 19.89 & $<0.05$ \\
\hline$\alpha_{\text {RLC }}$ & & & & $I_{\mathrm{k}(\mathrm{RLC})}$ & & & \\
\hline Temperature & 1 & 0.30 & $>0.05$ & Temperature & 1 & 1.90 & $>0.05$ \\
\hline Light & 1 & 6.93 & $<0.05$ & Light & 1 & 0.10 & $>0.05$ \\
\hline Temperature $\times$ light & 1 & 0.12 & $>0.05$ & Temperature $\times$ light & 1 & 11.09 & $<0.05$ \\
\hline$N P Q_{R L C}$ & & & & $\mathrm{ETR}_{\max (\mathrm{LC})}$ & & & \\
\hline Temperature & 1 & 60.90 & $<0.01$ & Temperature & 1 & 94.32 & $<0.001$ \\
\hline Light & 1 & 113.20 & $<0.01$ & Light & 1 & 25.08 & $<0.01$ \\
\hline Temperature $\times$ light & 1 & 177.04 & $<0.01$ & Temperature $\times$ light & 1 & 14.60 & $<0.01$ \\
\hline$\alpha_{\mathrm{LC}}$ & & & & $I_{\mathrm{k}(\mathrm{LC})}$ & & & \\
\hline Temperature & 1 & 0.20 & $>0.05$ & Temperature & 1 & 68.38 & $<0.01$ \\
\hline Light & 1 & 0.00 & $>0.05$ & Light & 1 & 14.62 & $<0.05$ \\
\hline Temperature $\times$ light & 1 & 0.57 & $>0.05$ & Temperature $\times$ light & 1 & 5.77 & $<0.05$ \\
\hline \multicolumn{8}{|l|}{$\mathrm{NPQ}_{\mathrm{LC}}$} \\
\hline Temperature & 1 & 9.39 & $<0.05$ & & & & \\
\hline Light & 1 & 28.40 & $<0.05$ & & & & \\
\hline Temperature $\times$ light & 1 & 19.52 & $<0.05$ & & & & \\
\hline
\end{tabular}


HT-HL, respectively. However, differences between all the treatments were not significant (Table 2). Compared to RLC, the $I_{\mathrm{k}}$ levels estimated from LC were relatively higher under the same treatment conditions. Additionally, the high temperature induced lower $I_{\mathrm{k}}$ regardless of light intensity as shown in the LC.

Fig. 2C,D illustrates typical responses of nonphotochemical quenching (NPQ) in RLC and LC to the temperature and light. Higher NPQs were found in LC, and the differences between RLC and LC were significant. The maximum NPQ in RLC was observed under LT-HL (Table 2), which was higher than those of other treatments (LT-LL, 34.1\% of LT-HL; HT-LL, $48.8 \%$ of LT-HL; and HT-HL, $41.5 \%$ of LT-HL). In LC, LT-HL induced the highest NPQ, while differences between other treatments were not significant.

Steady-state rapid light curve: The short-term photoacclimation and photoregulation were reflected by the responses of steady-state rapid light curve (SRLC) to ambient light (Fig. 3). The light responses of rETR $_{\max }$, SRLC in acclimated cultures under different temperature and light conditions were significantly different. In low temperature (LT-LL and LT-HL) treatments, rETR $_{\max , \text { SRLC }}$ increased with the increasing ambient light under PAR $<$ $463 \mu \mathrm{mol}$ (photon) $\mathrm{m}^{-2} \mathrm{~s}^{-1}$ to a maximum value, and then decreased under the light above the PAR value. In addition, the high light induced greater $\mathrm{rETR}_{\max }$ SRLC than that at the low light acclimation (Fig. $3 A$ ). Besides, in the hightemperature samples (HT-LL and HT-HL), maximum $\mathrm{rETR}_{\max , \text { SRLC }}$ were found at $322 \mu \mathrm{mol}$ (photon) $\mathrm{m}^{-2} \mathrm{~s}^{-1}$ and slightly decreased with the increasing light. However, the difference of $\mathrm{rETR}_{\max \text {, SRLC }}$ between high and low light was not significant, which is inconsistent to the responses of rETR $_{\max }$, SRLC to ambient light in low temperature acclimated cultures. The light responses of light-saturation $\left(I_{\mathrm{k}, \text { SRLC }}\right)$ showed that it increased almost linearly with the increasing PAR. In addition, LT-HL samples presented

Table 2. Changes of rapid light curves (RLC) and steady-state light curves (LC) parameters in Sargassum horneri cultured at different temperature and light. $\alpha$ - electron transport efficiency; $\mathrm{ETR}_{\max }-$ maximum electron transport rate; $I_{\mathrm{k}}-$ saturated irradiance; $\mathrm{NPQ}_{\max }$ - maximum nonphotochemical quenching; LT - low temperature; HT - high temperature; LL - low light; HL - high light. Values are means $\pm \mathrm{SD}, n=3$. Different lowercase letters indicate significant difference $(P<0.05)$ between the different treatments.

\begin{tabular}{llllll}
\hline Temperature & Light & $\alpha$ & ETR $_{\max }$ & $I_{\mathrm{k}}$ & $\mathrm{NPQ}_{\max }$ \\
\hline RLC & & & & \\
LT & LL & $0.28 \pm 0.00^{\mathrm{a}}$ & $38.62 \pm 2.27^{\mathrm{a}}$ & $135.96 \pm 21.51^{\mathrm{a}}$ & $0.42 \pm 0.03^{\mathrm{a}}$ \\
& HL & $0.26 \pm 0.04^{\mathrm{a}}$ & $41.95 \pm 4.25^{\mathrm{a}}$ & $164.26 \pm 6.15^{\mathrm{ad}}$ & $1.23 \pm 0.09^{\mathrm{b}}$ \\
HT & LL & $0.28 \pm 0.01^{\mathrm{a}}$ & $42.33 \pm 2.27^{\mathrm{a}}$ & $151.89 \pm 14.90^{\mathrm{ad}}$ & $0.60 \pm 0.05^{\mathrm{c}}$ \\
& HL & $0.24 \pm 0.01^{\mathrm{a}}$ & $30.52 \pm 1.32^{\mathrm{b}}$ & $127.25 \pm 8.36^{\mathrm{a}}$ & $0.51 \pm 0.04^{\mathrm{ac}}$ \\
LC & & & & \\
LT & LL & $0.26 \pm 0.01^{\mathrm{a}}$ & $56.73 \pm 4.85^{\mathrm{c}}$ & $215.68 \pm 17.53^{\mathrm{b}}$ & $2.08 \pm 0.39^{\mathrm{d}}$ \\
& HL & $0.27 \pm 0.02^{\mathrm{a}}$ & $72.10 \pm 3.13^{\mathrm{d}}$ & $265.64 \pm 21.55^{\mathrm{c}}$ & $4.10 \pm 0.57^{\mathrm{e}}$ \\
HT & LL & $0.28 \pm 0.03^{\mathrm{a}}$ & $46.47 \pm 1.68^{\mathrm{a}}$ & $168.59 \pm 19.11^{\mathrm{ad}}$ & $2.36 \pm 0.06^{\mathrm{d}}$ \\
& HL & $0.27 \pm 0.01^{\mathrm{a}}$ & $48.54 \pm 0.49^{\mathrm{a}}$ & $180.80 \pm 8.01^{\mathrm{bd}}$ & $2.55 \pm 0.17^{\mathrm{d}}$ \\
\hline
\end{tabular}

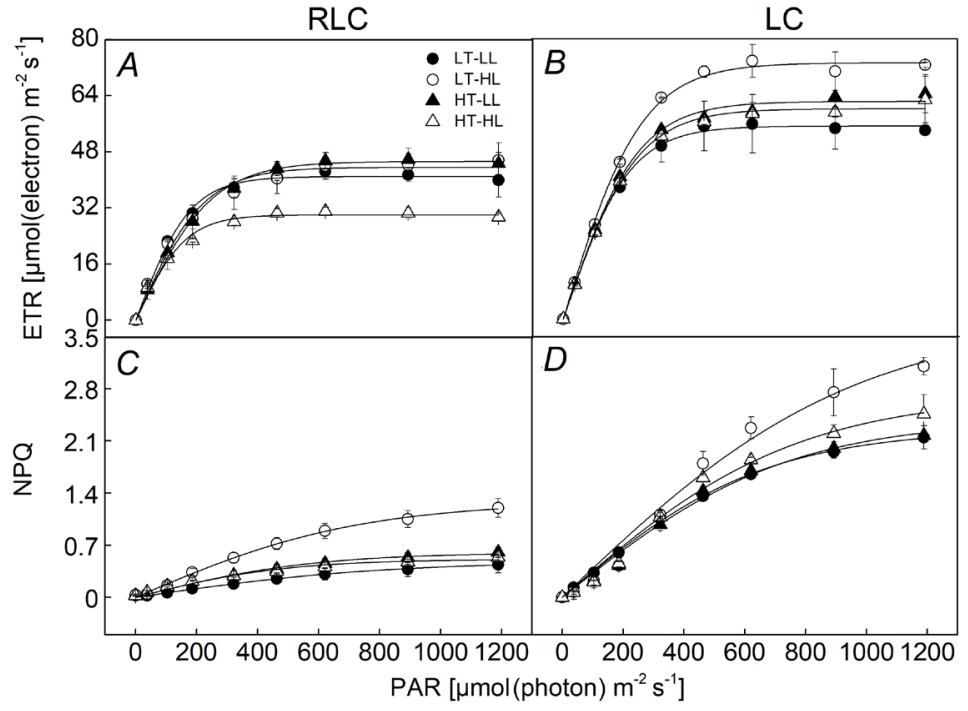

Fig. 2. Typical changes in relative electron transport rate (ETR) $(A, B)$ and nonphotochemical quenching (NPQ) $(C, D)$ as a function of photosynthetically active radiation (PAR) in Sargassum horneri cultured at different light and temperature. LT-LL - low temperature and low light; LT-HL - low temperature and high light; HT-LL - high temperature and low light; HT-HL - high temperature and high light; RLC - rapid light curves; LC - steady-state light curves. Values are means $\pm \mathrm{SD}, n=3$. 


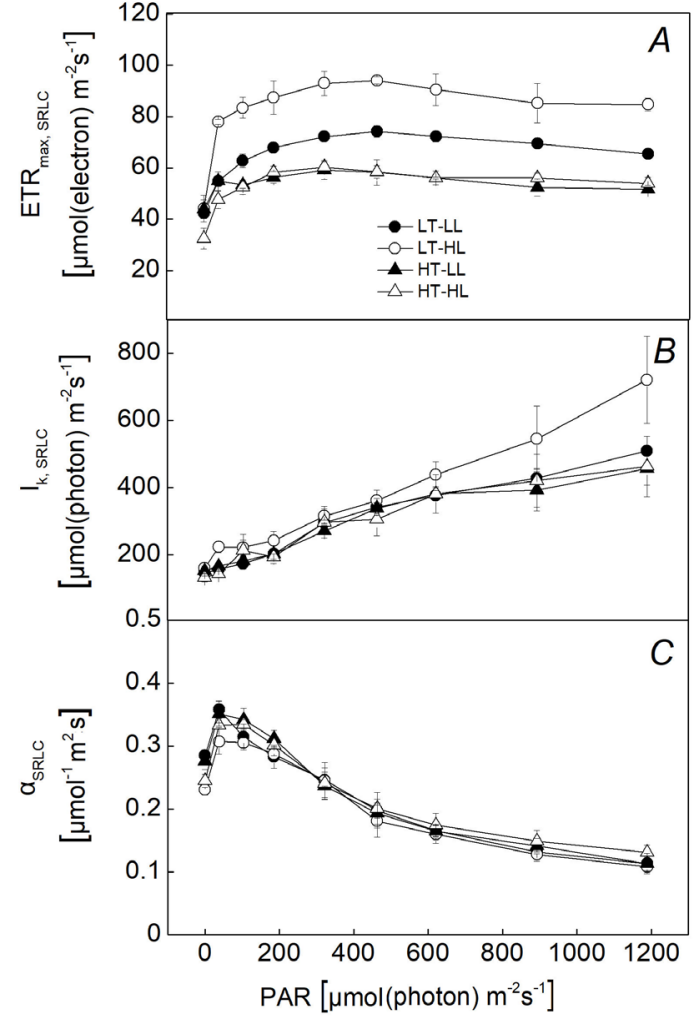

Fig. 3. Variation of SRLC (steady-state rapid light curves) parameters in Sargassum horneri cultured at different lights and temperatures. $\alpha_{\text {SRLC }}-$ electron transport efficiency; $\mathrm{ETR}_{\max , \text { SRLC }}$ - maximum electron transport rate; $I_{\mathrm{k}, \text { SRLC }}-$ saturated irradiance; LT-LL - low temperature and low light; LT$\mathrm{HL}$ - low temperature and high light; HT-LL - high temperature and low light; HT-HL - high temperature and high light. Values are means $\pm \mathrm{SD}, n=3$.

higher values of $I_{\mathrm{k}, \text { SRLC }}$ than those of other cultures. On the other hand, the electron transport efficiency of SRLC $\left(\alpha_{\text {SRLC }}\right)$ presented a biphasic pattern, with $\alpha_{\text {SRLC }}$ increasing under low light, until reaching the maximum value under $38 \mu \mathrm{mol}$ (photon) $\mathrm{m}^{-2} \mathrm{~s}^{-1}$, then decreased almost linearly with the increasing PAR, and the differences between all the treatments were not significant.

$\mathbf{Y}_{(\mathrm{II})}, \mathbf{Y}_{(\mathrm{NO})}$, and $\mathbf{Y}_{(\mathrm{NPQ})}$ vs. PAR curves: The effective quantum yield of PSII [ $\left.\mathrm{Y}_{(\mathrm{II})}\right]$ markedly decreased with the ambient light intensity (Fig. 4A,B). The rapid light curve (RLC) shows that the higher light intensity and higher temperature, the greater decrease of $\mathrm{Y}_{\text {(II) }}$. However, as shown in the steady-state light curve (LC), the high light resulted in a greater $Y_{(\text {II }}$ than that of low light did under low temperature. Under a high temperature, the difference of $\mathrm{Y}_{\text {(II) }}$ between high and low light treatments was not significant.

The responses of quantum yield of nonregulated energy dissipation $\left[\mathrm{Y}_{(\mathrm{NO})}\right]$ to the ambient light intensity varied with the increase in temperature and light intensity (Fig. 4C,D). Compared to RLC, relatively lower $\mathrm{Y}_{(\mathrm{NO})}$ is seen in LC. As shown in $\mathrm{RLC}, \mathrm{Y}_{(\mathrm{NO})}$ increased quickly at the beginning under low light and then slowly reached a maximal value and stayed on. Besides, $\mathrm{Y}_{(\mathrm{NO})}$ under LT-HL treatment was lower than those of other treatments (LT-LL, HT-LL, and HT-HL), while no differences were found between these treatments. In $\mathrm{LC}, \mathrm{Y}_{(\mathrm{NO})}$ decreased with the ambient light intensity under LT-HL, HT-LL, and HT-HL conditions, while the $\mathrm{Y}_{(\mathrm{NO})}$ of LT-LL showed no change. In addition, the responses of $\mathrm{Y}_{(\mathrm{NO})}$ to ambient light intensity under LT-HL treatment were smaller than those of other treatments (LT-LL, HT-LL, and HT-HL).

The light response of quantum yield about regulated energy dissipation $\left[\mathrm{Y}_{(\mathrm{NPQ})}\right]$ in RLC and LC varied markedly (Fig. 4E,F). In RLC, LT-HL could apparently increase the $\mathrm{Y}_{(\mathrm{NPO})}$. On the other hand, no differences were found between other treatments (LT-LL, HT-LL, and HT-HL). In $\mathrm{LC}$, the light response of $\mathrm{Y}_{(\mathrm{NPQ})}$ presented an increase with the ambient light intensity, while the differences between all treatments were minor.

$\Delta \boldsymbol{\alpha}$ vs. NPQ: A linear relationship was observed between NPQ and the high light-induced decrease of $\Delta \alpha$ under low light for each light group (Fig. 5; Serôdio et al. 2006). NPQ was the NPQ measured under the steady state for $S$. horneri. $\Delta \alpha=\alpha_{\mathrm{m}}-\alpha$, where $\alpha_{\mathrm{m}}$ is the maximum value of $\alpha_{\mathrm{m}} v s$. PAR. Correlations between $\Delta \alpha$ and NPQ were highly significant in all cases. The difference of slopes of the regressions of $\Delta \alpha$ on NPQ calculated from the different cultivations were significant $\left(\mathrm{F}_{3,8}=140.935, P<0.001\right.$; ANOVA test for homogeneity of slopes).

\section{Discussion}

Under various environmental factors, short-term photosynthetic acclimation to ambient light and temperature is an important photoprotective way by which the light energy capture and metabolic energy consumption were balanced via effective energy distribution (Davison and Pearson 1996, Ensminger et al. 2005). Photoacclimation could be realized by changing the size or the number of photosynthetic units (Williamson et al. 2018). Under a suitable growth temperature, low light (LL) acclimation of $S$. horneri could increase the number of photosynthetic unites to enhance the light-utilization efficiency $(\alpha)$ but had low $\mathrm{rETR}_{\max }$ as illustrated in the rapid light curve (RLC), which is similar to the results of Beer et al. (2014). Conversely, in the steady-state light curve (LC), the $\alpha$ values between LL and HL showed no difference, implying that the size of photosynthetic units was changed by HL. The significantly higher $\mathrm{rETR}_{\max }$ indicated that photoacclimation could be achieved mainly by changing the size of photosynthetic units and enhancing the light energy utilization to prevent the photodamage to PSII under HL conditions (Williamson et al. 2018).

PSII is known as one of the most thermosensitive components of photosynthetic apparatus (Havaux 1996). Under a high temperature stress, the fluidity of thylakoid membranes could be increased, causing the lightharvesting complexes of PSII dislodge from thylakoid membrane (Mathur et al. 2014). It was reported that the reasons for inhibition of PSII caused by high temperature 


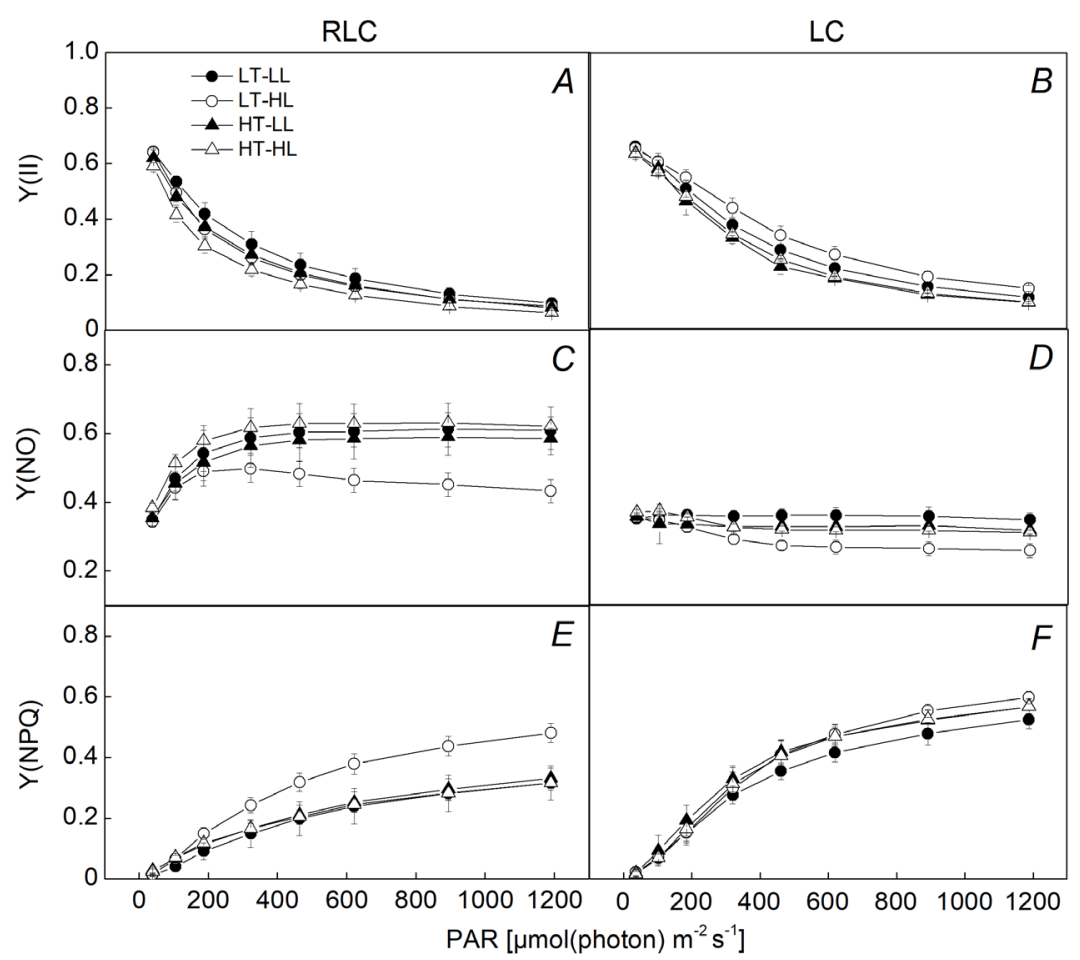

Fig. 4. Changes in effective PSII quantum yield $\left[\mathrm{Y}_{(\mathrm{II})}\right](A, B)$, nonregulated energy dissipation $\left[\mathrm{Y}_{(\mathrm{NO})}\right](C, D)$, and regulated energy dissipation $\left[\mathrm{Y}_{(\mathrm{NPQ})}\right](E, F)$ as a function of photosynthetically active radiation (PAR) in Sargassum horneri cultured at a different light and temperature. LT-LL - low temperature and low light; LT-HL - low temperature and high light; HT-LL - high temperature and low light; HT-HL - high temperature and high light; RLC - rapid light curve; LC - steady-state light curve. Values are means $\pm \mathrm{SD}, n=3$.

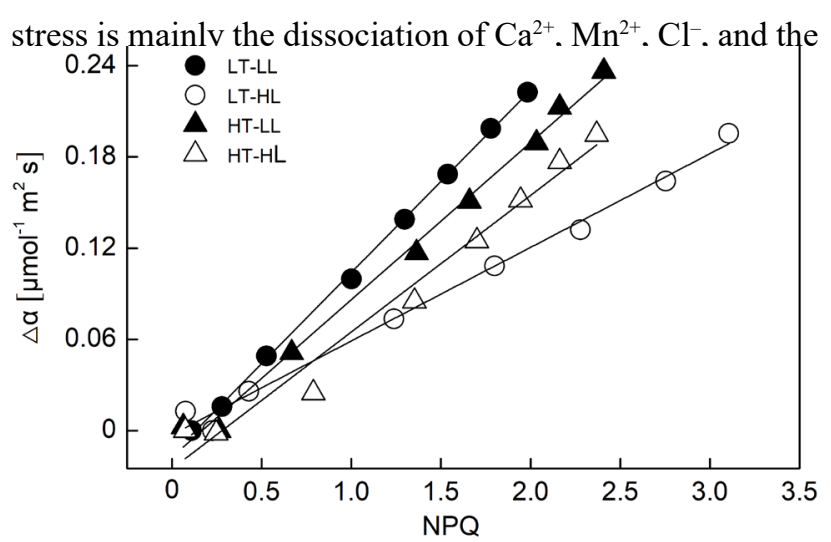

Fig. 5. Linear relationship between $\Delta \alpha$ and NPQ (measured under steady state) for Sargassum horneri cultured at different conditions of temperature and light. LT-LL - low temperature and low light; LT-HL - low temperature and high light; HT-LL high temperature and low light; HT-HL - high temperature and high light.

release of extrinsic 18, 24, and 33-kDa polypeptides from oxygen-evolving complex (OEC) (Yang et al. 2018). The PSII inhibition caused by high temperature stress was also affected by light (Yan et al. 2013). In the present study, high light intensity promoted the inhibition of $\mathrm{rETR}_{\max }$ in $S$. horneri at high temperature. As shown in the RLC, the $\mathrm{rETR}_{\max }$ of high temperature and high light acclimated treatments was significantly smaller than those of other treatments, suggesting that the electron transport at the PSII acceptor side was inhibited (Yang et al. 2018). In addition, as shown in the LC, high light treatment produced the highest $\mathrm{rETR}_{\max }$ under low temperature, reflecting the actual photosynthetic performance of $S$. horneri. Therefore, it is likely that the high temperature stress was the predominant factor for the inhibition of PSII.

To avoid the photodamage to PSII, plants have evolved a series of tolerance mechanisms to adapt stresses, including ion transporters, induction of antioxidant defense, and accumulation of osmoprotectants (Mathur et al. 2014). In case of a sudden stress, nonphotochemical quenching (NPQ) is a fast and flexible photoprotective mechanism to avoid the damage to the photosynthetic apparatus by dissipating excess absorbed light energy (Müller et al. 2001). Higher capacity of NPQ means generally a better adaptation to high light (Pniewski et al. 2017). As shown in Fig. $2 \mathrm{C}$ and Table 2, the LT-HL acclimation resulted in the highest NPQ value in all cases, suggesting that the greater size of XC pigments pool $(\Sigma X C)$ was accumulated in LT-HL after 30 -min dark treatments, where $\Sigma \mathrm{XC}$ is the sum of violaxanthin (VX) plus antheraxanthin and zeaxanthin $(Z X)$. Because NPQ depends on the size and de-epoxidation state of $\Sigma \mathrm{XC}$, a greater $\Sigma \mathrm{XC}$ would result in a faster NPQ induction (Ocampo-Alvarez et al. 2013). Additionally, in the LC, high light condition induced greater NPQ, especially under low temperature, while high temperature or low light caused lower NPQ, and the difference between them was not significant, implying that high temperature reduced the size of $\Sigma \mathrm{XC}$ but induced a faster VX to $\mathrm{ZX}$ conversion. If excess energy exceeds the photochemical and nonphotochemical capacities, the reactive oxygen species (ROS) could be produced, which would damage the PSII reaction centers (Wilson et al. 2006). A high quantum yield of nonregulated energy dissipation $\left[\mathrm{Y}_{(\mathrm{NO})}\right]$ is often used to indicate that both photochemical and nonphotochemical capacity are 
inefficient (Hendrickson et al. 2004, Wang et al. 2009). In both RLC and LC (Fig. 4), a lower $\mathrm{Y}_{(\mathrm{NO})}$ was found at LT-HL, implying that high light enhanced the photochemical and nonphotochemical capacity. The $\mathrm{Y}_{(\mathrm{NO})}$ values in all the treatments showed a marked increase in RLC, while relatively stable $\mathrm{Y}_{(\mathrm{NO})}$ occurred in LC, suggesting that the rapid strong light induced more ROS production and lowered the photochemical and nonphotochemical capacity. A high $\mathrm{Y}_{(\mathrm{NPQ})}$ means that the light intensity is excessive, but the plant has the capacity to protect itself (Wang et al. 2009). In the RLC, we found that high temperature could destroy the capacity of protection, and no difference was observed between low and high light treatments, while high light showed a better regulation than the low light did under low temperature conditions. Therefore, high temperature was the main factor of destructing the capacity of regulation, and the low light was harmful, too. On the other hand, in the LC, it is easily to find that the $\mathrm{Y}_{(\mathrm{NPQ})}$ showed the same pattern to that in the RLC, while $\mathrm{Y}_{(\mathrm{NPQ})}$ in the LC was higher than that in the RLC. All these clearly indicate that the PSII reaction centers (RCIIs) stay completely open and the photosynthetic yield is determined largely by the changes in NPQ in LC (Kramer et al. 2004). Conversely, the rapid strong light could cause the closure of RCIIs during RLC. The reasons for differences between LC and RLC may be that there is no fast phase of NPQ ( $\triangle \mathrm{pH}$-dependent quenching) in brown algae, resulting in slow NPQ induction (GarcíaMendoza and Colombo-Pallotta 2007, García-Mendoza et al. 2011). The lasting time in RLC is relatively short compared to that in LC, it may not be enough to induce the NPQ generation completely $\left(\mathrm{NPQ}_{\mathrm{RLC}}<\mathrm{NPQ} \mathrm{LC}_{\mathrm{L}}\right)$. Therefore, excess absorbed light energy could lead to the overreduction of $\mathrm{Q}_{\mathrm{A}}$ as well as closure of the RCIIs and the quenching of reaction center due to nonradiative charge recombination between $\mathrm{Q}_{\mathrm{A}}^{-}$and the primary donor of PSII (Ivanov et al. 2008). With the illumination time lasting, high light increased the electron turnover through the PSII and activated the Calvin-Benson cycle enzyme (Pniewski et al. 2018), so that the reaction center could gradually be opened again. Meanwhile, NPQ gradually appeared during light, so $\mathrm{Y}_{(\mathrm{NPQ})}$ rises, while $\mathrm{Y}_{(\mathrm{NO})}$ remains stable. This suggests that $S$. horneri relies more on 'nonregulated' $\mathrm{Y}_{(\mathrm{NO})}$ than on 'regulated' $\mathrm{Y}_{(\mathrm{NPQ})}$ energy dissipation for photoprotection $\left[\mathrm{Y}_{(\mathrm{NO})}>\mathrm{Y}_{(\mathrm{NPQ})}\right.$ ] in RLC during exposing to short periods of high light; while during a long duration of high light, NPQ is main mechanism to deal with the excess absorbed light energy.

It is reported that the inhibition of $\alpha_{\mathrm{RLC}}$ under high light stress is most likely caused by the operation of reversible photoprotective NPQ processes (Serôdio et al. 2006). The conversion of VX to ZX takes place under high light and the back conversion takes place in darkness or under low light intensity (Ocampo-Alvarez et al. 2013). The accumulation of $\mathrm{ZX}$ decreases the transfer efficiency of absorbed energy to RCIIs (Serôdio et al. 2006), which is supported by the fact that the tight correlations between $\Delta \alpha_{\text {SRLC }}$ and NPQ were found in all cases. As shown in Fig. 5 , the $\Delta \alpha_{\mathrm{SRLC}}$ of low light acclimation was more sensitive to NPQ than that of high light acclimation, indicating that the transfer efficiency of absorbed energy to the RCIIs under low light acclimation treatments was more likely affected by high light stress, and high light acclimation had a better photoprotective mechanism (larger $\Sigma \mathrm{XC}$ ) to avoid photodamage under a high light stress. Additionally, as shown in Fig. 5, high temperature enhanced the sensitivity of $\Delta \alpha_{\text {SRLC }}$ to NPQ under high light acclimation treatments, which is in coincidence with the fact that high temperature reduced the size of $\Sigma \mathrm{XC}$.

In conclusion, we illustrated the short-term photoacclimation and photoregulation strategies of $S$. horneri in responses to temperature and light changes. $S$. horneri was adapted to grow under low temperature and high light conditions. High temperature was the predominant factor for causing the inhibition of PSII, while high light could improve the photoprotective ability. Under the global warming scheme, improving the water quality in coastal areas is an effective measure to protect Sargassum beds.

\section{References}

Beer S., Björk M., Beardall J.: Photosynthesis in the Marine Environment. Pp. 157-173. Wiley-Blackwell, Hoboken 2014. Belshe E.F., Durako M.J., Blum J.E.: Photosynthetic rapid light curves (RLC) of Thalassia testudinum exhibit diurnal variation. - J. Exp. Mar. Biol. Ecol. 342: 253-268, 2007.

Bi Y., Zhang S., Wang W., Wu Z.: [Vertical distribution pattern of Sargassum horneri and its relationship with environmental factors around Gouqi Island.] - Acta Ecol. Sin. 34: 49314937, 2014. [In Chinese]

Bilger W., Björkman O.: Role of the xanthophyll cycle in photoprotection elucidated by measurements of light-induced absorbance changes, fluorescence and photosynthesis in leaves of Hedera canariensis. - Photosynth. Res. 25: 173185,1990

Davison I.R., Pearson G.A.: Stress tolerance in intertidal seaweeds. - J. Phycol. 32: 197-211, 1996.

Ensminger I., Foerster J., Hagen C., Braune W.: Plasticity and acclimation to light reflected in temporal and spatial changes of small-scale macroalgal distribution in a stream. - J. Exp. Bot. 56: 2047-2058, 2005.

Esteban R., Martínez B., Fernández-Marín B. et al.: Carotenoid composition in Rhodophyta: insights into xanthophyll regulation in Corallina elongata. - Eur. J. Phycol. 44: 221230, 2009.

Gao G., Jin P., Liu N. et al.: The acclimation process of phytoplankton biomass, carbon fixation and respiration to the combined effects of elevated temperature and $\mathrm{pCO}_{2}$ in the northern South China Sea. - Mar. Pollut. Bull. 118: 213-220, 2017.

García-Mendoza E., Colombo-Pallotta M.F.: The giant kelp Macrocystis pyrifera presents a different nonphotochemical quenching control than higher plants. - New Phytol. 173: 526-536, 2007.

García-Mendoza E., Ocampo-Alvarez H., Govindjee.: Photoprotection in the brown alga Macrocystis pyrifera: Evolutionary implications. - J. Photoch. Photobio. B. 104: 377-385, 2011.

Harley C.D.G., Anderson K.M., Demes K.W. et al.: Effects of climate change on global seaweed communities. - J. Phycol. 48: 1064-1078, 2012.

Havaux M.: Short-term responses of PSI to heat stress. Induction of a PS II-independent electron transport through PS I fed by stromal components. - Photosynth. Res. 47: 85-97, 1996.

Hendrickson L., Furbank R.T., Chow W.S.: A simple alternative 
approach to assessing the fate of absorbed light energy using chlorophyll fluorescence. - Photosynth. Res. 82: 73, 2004.

IPCC 2013: Climate Change 2013: The Physical Science Basis. In: Stocker T.F., Qin D., Plattner G.-K. et al. (ed.): Working Group I Contribution to the Fifth Assessment Report of the Intergovernmental Panel on Climate Change. Cambridge University Press, New York 2013.

Ivanov A.G., Hurry V., Sane P.V. et al.: Reaction centre quenching of excess light energy and photoprotection of photosystem II. J. Plant Biol. 51: 85, 2008.

Jassby A.D., Platt T.: Mathematical formulation of the relationship between photosynthesis and light for phytoplankton. Limnol. Oceanogr. 21: 540-547, 1976.

Koch M., Bowes G., Ross C., Zhang X.H.: Climate change and ocean acidification effects on seagrass and marine macroalgae. - Glob. Change Biol. 19: 103-132, 2013.

Komatsu T., Ariyama H., Nakahara H., Sakamoto W.: Spatial and temporal distributions of water temperature in a Sargassum forest. - J. Oceanogr. 38: 63-72, 1982.

Komatsu T., Fukuda M., Mikami A. et al.: Possible change in distribution of seaweed, Sargassum horneri, in northeast Asia under A2 scenario of global warming and consequent effect on some fish. - Mar. Pollut. Bull. 85: 317-324, 2014.

Kramer D.M., Johnson G., Kiirats O., Edwards G.E.: New fluorescence parameters for the determination of $\mathrm{Q}_{\mathrm{A}}$ redox state and excitation energy fluxes. -Photosynth. Res. 79: 209, 2004.

Lavaud J., Lepetit B.: An explanation for the inter-species variability of the photoprotective non-photochemical chlorophyll fluorescence quenching in diatoms. - BBABioenergetics 1827: 294-302, 2013.

Li X., Zhang Q., He J. et al.: Photoacclimation characteristics of Sargassum thunbergii germlings under different light intensities. - J. Appl. Phycol. 26: 2151-2158, 2014.

Mathur S., Agrawal D., Jajoo A.: Photosynthesis: Response to high temperature stress. - J. Photoch. Photobio. B 137: 116$126,2014$.

Miki O., Okumura C., Tuji K., Takami M.: Effects of preservation period of fertilized eggs and high concentrations of nitrogen in nutrient sources on germling growth of Sargassum horneri.J. Appl. Phycol. 28: 2883-2890, 2016.

Müller P., Li X.P., Niyogi K.K.: Non-photochemical quenching. A response to excess light energy. - Plant Physiol. 125: 15581566, 2001.

Murakami K., Yamaguchi Y., Noda K. et al.: Seasonal variation in the chemical composition of a marine brown alga, Sargassum horneri (Turner) C. Agardh. - J. Food Compos. Anal. 24: 231-236, 2011

Ocampo-Alvarez H., Garcia-Mendoza E., Govindjee: Antagonist effect between violaxanthin and de-epoxidated pigments in nonphotochemical quenching induction in the $\mathrm{qE}$ deficient brown alga Macrocystis pyrifera. - BBA-Bioenergetics 1827: 427-437, 2013

Pang S.J., Liu F., Shan T.F. et al.: Cultivation of the brown alga Sargassum horneri: Sexual reproduction and seedling production in tank culture under reduced solar irradiance in ambient temperature. - J. Appl. Phycol. 21: 413-422, 2009.

Pniewski F.F., Richard P., Latała A., Blanchard G.: Nonphotochemical quenching in epipsammic and epipelic microalgal assemblages from two marine ecosystems. - Cont. Shelf Res. 136: 74-82, 2017.

Pniewski F.F., Richard P., Latała A., Blanchard G.: Long- and short-term photoacclimation in epipsammon from non- tidal coastal shallows compared to epipelon from intertidal mudflat. - J. Sea Res. 136: 1-9, 2018.

Ralph P.J., Gademann R.: Rapid light curves: a powerful tool to assess photosynthetic activity. - Aquat. Bot. 82: 222-237, 2005.

Sanjeewa K.K.A., Fernando I.P.S., Kim E.A. et al.: Antiinflammatory activity of a sulfated polysaccharide isolated from an enzymatic digest of brown seaweed Sargassum horneri in RAW 264.7 cells. - Nutr. Res. Pract. 11: 3-10, 2017.

Serôdio J., Lavaud J.: A model for describing the light response of the nonphotochemical quenching of chlorophyll fluorescence. - Photosynth. Res. 108: 61-76, 2011.

Serôdio J., Vieira S., Cruz S., Coelho H.: Rapid light-response curves of chlorophyll fluorescence in microalgae: Relationship to steady-state light curves and non-photochemical quenching in benthic diatom-dominated assemblages. - Photosynth. Res. 90: 29-43, 2006.

Stirbet A., Govindjee: On the relation between the Kautsky effect (chlorophyll $a$ fluorescence induction) and photosystem II: basics and applications of the OJIP fluorescence transient. J. Photoch. Photobio. B 104: 236-257, 2011.

Sun J., Zhuang D., Chen W. et al.: [Studies on sexual reproduction and seedling production of the brown alga Sargassum horneri.] - South China Fish. Sci. 4: 6-14, 2008. [In Chinese]

Sun J.Z., Ning X.R., Le F.F. et al.: Long term changes of biodiversity of benthic macroalgae in the intertidal zone of the Nanji Islands. - Acta Ecol. Sin. 30: 106-112, 2010.

Terawaki T., Yoshikawa K., Yoshida G. et al.: Ecology and restoration techniques for Sargassum beds in the Seto Inland Sea, Japan. - Mar. Pollut. Bull. 47: 198-201, 2003.

Wang L.J., Loescher W., Duan W. et al.: Heat acclimation induced acquired heat tolerance and cross adaptation in different grape cultivars: Relationships to photosynthetic energy partitioning. - Funct. Plant Biol. 36: 516-526, 2009.

White A.J., Critchley C.: Rapid light curves: A new fluorescence method to assess the state of the photosynthetic apparatus. Photosynth. Res. 59: 63-72, 1999.

Williamson C.J., Perkins R., Yallop M.L. et al.: Photoacclimation and photoregulation strategies of Corallina (Corallinales, Rhodophyta) across the NE Atlantic. - Eur. J. Phycol. 53: 290-306, 2018.

Wilson K.E., Ivanov A.G., Öquist G. et al:: Energy balance, organellar redox status, and acclimation to environmental stress. - Can. J. Bot. 84: 1355-1370, 2006.

Yamamoto Y.: Quality control of photosystem II: the mechanisms for avoidance and tolerance of light and heat stress are closely linked to membrane fluidity of the thylakoids. - Front. Plant Sci. 7: 1136, 2016.

Yan K., Chen P., Shao H. et al.: Dissection of photosynthetic electron transport process in sweet sorghum under heat stress. PLoS ONE 8: e62100, 2013.

Yang X.Q., Zhang Q.S., Zhang D. et al.: Interaction of high seawater temperature and light intensity on photosynthetic electron transport of eelgrass (Zostera marina L.). - Plant Physiol. Bioch. 132: 453-464, 2018.

Yu J., Li J., Wang Q. et al.: Growth and resource accumulation of drifting Sargassum horneri (Fucales, Phaeophyta) in response to temperature and nitrogen supply. - J. Ocean Univ. China 18: 1216-1226, 2019.

Zhang S.Y., Wang L., Wang W.D.: Algal communities at Gouqi Island in the Zhoushan archipelago, China. - J. Appl. Phycol. 20: 853-861, 2008.

(C) The authors. This is an open access article distributed under the terms of the Creative Commons BY-NC-ND Licence. 